\title{
Burnout among obstetrics and gynaecology registrars in teaching hospitals of the University of the Witwatersrand Medical School
}

\author{
G M Balie, MB ChB, Dip Obs, FCOG (SA), MMed (O\&G); S J Branch, MB BCh, FCOG (SA), MMed (O\&G); \\ H A du Toit Lombaard, MB ChB, FCOG (SA), MMed (O\&G), Cert Maternal and Fetal Medicine, PG Dip HSE
}

Department of Obstetrics and Gynaecology, School of Clinical Medicine, Faculty of Health Sciences, University of Witwatersrand, Johannesburg, South Africa

Corresponding author: G M Balie (Gaynor.balie16@gmail.com)

\begin{abstract}
Background. There is an upward trend in burnout among all professions, including healthcare workers. Few causative factors have been identified and few interventions are available to decrease risk. At present, there are no data on burnout among obstetricians and gynaecologists in South Africa.

Objectives. To determine the prevalence of burnout among registrars in the department of Obstetrics and Gynaecology the University of the Witwatersrand and identify risk factors for burnout in this setting.

Methods. A cross-sectional study was conducted, using a convenience sample of registrars at the University of the Witwatersrand Obstetrics and Gynaecology circuit between September and October 2017. Electronic surveys were sent to 61 registrars in the circuit at that time. The Maslach Burnout Inventory was used to evaluate burnout for each of the three burnout subgroups both separately and cumulatively.

Results. Of the responding participants, $78 \%$ were women. All four years of training were represented with $\sim 23 \%$ of respondents in each year of study. Only 6\% showed burnout in all three subgroups. There were high levels both of emotional exhaustion (85\%) and depersonalisation (78.7\%). Significant predictors of emotional exhaustion included female gender and number of years as a medical officer before becoming a registrar.

Conclusion. Burnout, particularly among women, is significantly high among registrars in the O\&G department at University of the Witwatersrand. Increased awareness and support for those affected is vital to improve registrar functioning and resultant patient care.
\end{abstract}

S Afr J Obstet Gynaecol 2019;25(3):75-78 https://doi.org/10.1796/SAJOG.2019.v25i3.1490

Obstetrics and Gynaecology (O\&G) is an increasingly stressful field to work in owing to increased litigation and workload. ${ }^{[1]}$ Burnout is increasing among healthcare workers, including registrars in $\mathrm{O} \& \mathrm{G},{ }^{[2]}$ which discourages junior doctors from specialising in the field. Possible risk factors associated with burnout include patient load, long working hours, poor supervision and the amount of after-hour duties. ${ }^{[2]}$ Registrars have large workloads, often with limited resources and staffing. Registrars in O\&G are expected to perform well academically in addition to providing obstetrical and gynaecological services during their training. To our knowledge, there are no studies that have assessed burnout among $O \& G$ in South Africa (SA). At the time of writing, studies of burnout in the medical profession in SA are limited to 3 main studies: (i) a study of paediatric medical officers in Cape Town; ${ }^{[3]}$ (ii) a study of medicalrelated professionals in public hospitals in Bloemfontein ${ }^{[4]}$ and (iii) a study of anaesthetists at the University of the Witwatersrand circuit, and the private sector in Johannesburg. ${ }^{[5]}$ Chronic stress and burnout can result from adverse working conditions, high patient load and the need to make critical decisions that optimise patient outcome. These factors can be particularly challenging in obstetrics where the wellbeing of both mother and baby is involved. Emotional and physical stress can negatively impact patient management, the healthcare worker's quality of life and mental health, resulting in reduced productivity and an increased risk of medical errors. ${ }^{[6]}$
The Maslach Burnout Inventory (MBI) is currently the standard test used in medical literature to characterise burnout. ${ }^{[7]}$ The MBI scale consists of 22 items, subdivided into three groups: (i) emotional exhaustion; (ii) depersonalisation; and (iii) reduced personal accomplishment. ${ }^{[7]}$ All items are framed as statements about personal attitudes or emotions. ${ }^{[7]}$ The frequencies of these feelings are measured and an individual score is assigned. Inverse grading is used to measure low personal accomplishment: the lower the score, the greater the feelings of low personal accomplishment. The authors of the MBI have identified six main areas that lead to burnout: (i) workload; (ii) doing a specific job; (iii) control and decision-making; (iv) recognition for the job done; $(v)$ fairness in the job and values of the company worked for; and (vi) community. When any of these are not in keeping with the healthcare worker's values, it can lead to burnout. ${ }^{[7]}$

\section{Methods \\ Data collection}

An analytical cross-sectional study, using a convenience sample, was conducted between September and October 2017, by means of an electronic survey. All registrars and supernumerary registrars in the University of the Witwatersrand O\&G circuit were eligible, if registered with the Health Professions Council of SA (HPCSA) between September and October 2017. The survey had two parts: 
(i) demographic information about the participants; (ii) the MBI health-services survey. A list of the 61 current registrars was obtained from the department and the survey was sent to each registrar, who replied anonymously. The deidentified responses were submitted via LimeSurvey software package (Schmitz, Germany) and forwarded to the principal researcher.

\section{Data analysis}

Demographic information for each participant on the LimeSurvey section was identified by number only and sent to MindGarden (MindGarden Inc., California) for the MBI section of the survey. Once assessed, data were returned to the principal researcher for additional analysis. Data were inputted in Excel and exported into STATA (StatCorp., USA). Burnout data were derived from a Likert scale and frequencies and corresponding percentages recorded for each category on the scale. Relationships between categorical variables were analysed using the $\chi^{2}$ test. Risk factors were calculated with ordinal regression. Continuous data, such as the burnout score, were assessed for normality and, where appropriate, presented as means and standard deviations. Precision was verified using the $95 \%$ confidence interval (CI) and $p$-values $<0.05$ were regarded as significant.

\section{Study approval and ethics}

Permission for the study was obtained from the Head of School of Clinical Medicine of the University of the Witwatersrand. Study approval was sought from the University of the Witwatersrand Human Research Ethics Committee (ref. no. M170701) before data collection commenced. An information sheet along with the survey was provided to the registrars, detailing the nature of the study and specifying that participant consent was given by answering and submitting the survey. Participants were made aware of the resources provided by The South African Depression and Anxiety Group (SADAG) in the information sheet.

\section{Results \\ Participant demographics}

Burnout data were derived from 47 registrars (77\%). The majority were SA citizens $(80.8 \%)$ and female (78.7\%). This sample size was representative of the current registrar group at the University of the Witwatersrand's department of O\&G. Just under half (48.9\%) were 31 - 35 years. Over half $(59.5 \%)$ the study participants were married, $10(21.3 \%)$ reported being in a stable relationship and 9 (19.2\%) were single. Twenty-five (53.2\%) reported they had children. Thirty-five $(74.5 \%)$ said they had a support system in Johannesburg. Twenty study participants (42.5\%) reported they had failed an examination related to their specialist training. Of these 20 participants, 9 (19.2\%) reported that the failure had affected their confidence. One $(2.1 \%)$ person reported that they were not financially independent, and an additional participant was reliant on funding from elsewhere. Half the study participants (55.0\%) reported experiencing a recent loss or event that negatively affected their personal lives. There was an even distribution between the number of years of study with $11(23.4 \%)$ in their first year, $14(23.8 \%)$ in their second year, and $11(23.4 \%)$ in either their third year or fourth year. A total of $36(76.6 \%)$ study participants reported having worked as medical officers (MO) prior to starting as registrars in this department. Twenty-nine (65.9\%) of these participants had been MOs for $<5$ years, 4 (9.1\%) for 5 - 10 years and $3(6.8 \%)$ for $>10$ years (Fig. 1$)$.

\section{Burnout among registrars in Obstetrics and Gynaecology}

Fig. 2 shows the distribution of emotional exhaustion, depersonalisation and personal accomplishment among the study participants. Forty $(85.1 \%)$ reported high emotional exhaustion, while $6(12.8 \%)$ reported low emotional exhaustion. Thirty-seven (78.7\%) reported high levels of depersonalisation, $6(12.8 \%)$ reported average depersonalisation and 4 (8.5\%) had low depersonalisation. Just under half (48.9\%) of the participants reported low personal accomplishment. Fourteen (28.8\%) participants reported average personal accomplishment, while $10(21.3 \%)$ reported high personal accomplishment. Fig. 3 shows the number of study participants with high levels of two or more of the outcomes. Three participants (6.4\%) had high emotional exhaustion, high depersonalisation and low personal accomplishment. Thirty-four (72.3\%) participants had both high emotional exhaustion and high depersonalisation. Six (12.8\%) had high emotional exhaustion and low personal accomplishment. Four (8.5\%) had high depersonalisation and low personal accomplishment. Significant predictors of high emotional exhaustion were female gender and number of years spent as a MO prior to the training programme. The odds of emotional exhaustion were 2 times higher in females than males (OR 2.11; 95\% CI 0.38 - 3.85; $p=0.02$ ). The odds of emotional exhaustion increased 3 times if the study participant had been an MO for $<10$ years (OR 3.04; 95\% CI $0.34-5.74 ; p=0.03$ ). Age was associated

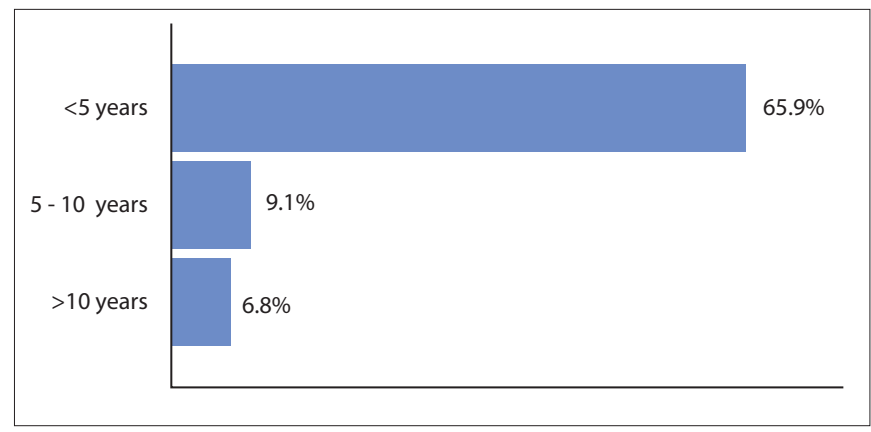

Fig. 1. Number of years working as a medical officer prior to being a registrar.

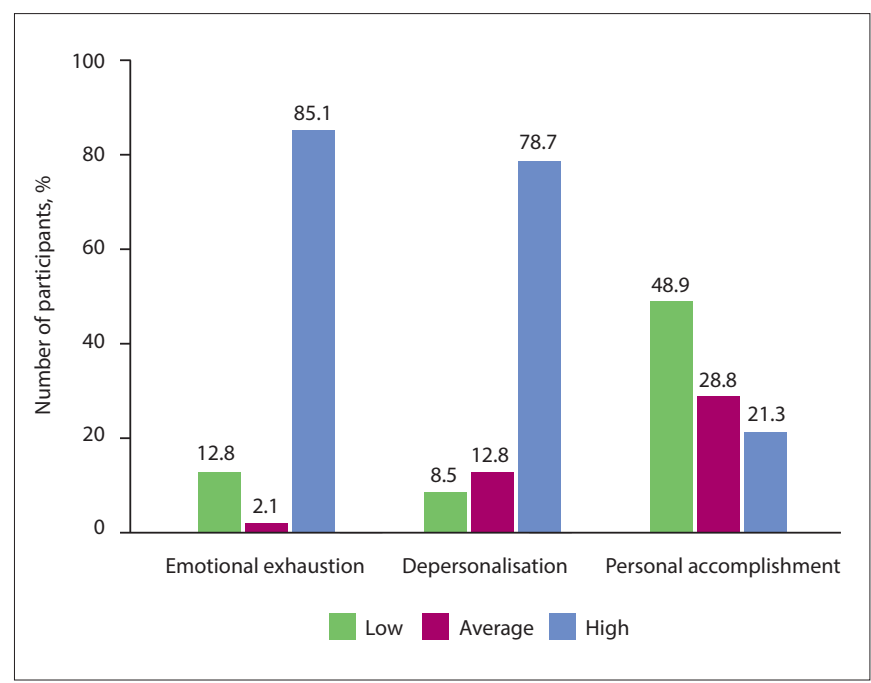

Fig. 2. Frequencies in the levels of emotional exhaustion, depersonalisation and personal accomplishment among study participants. 


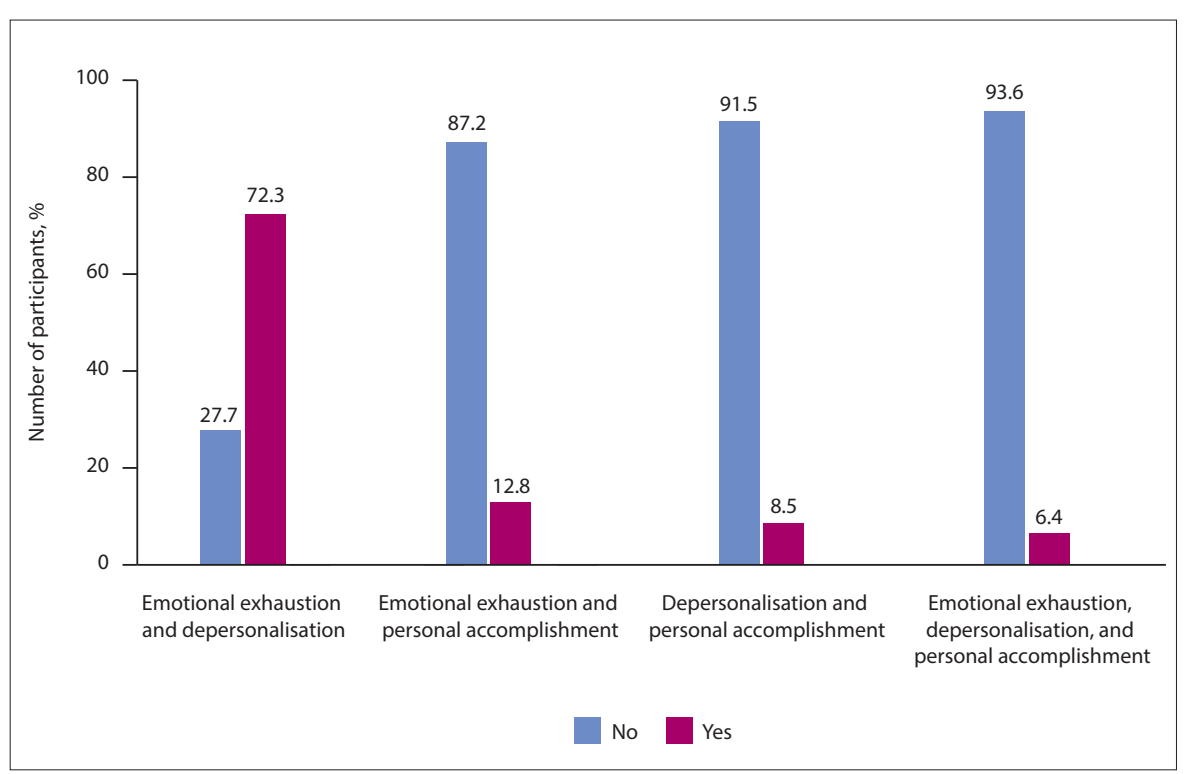

Fig. 3. Frequencies of two or more outcomes among study participants.

with high depersonalisation $(p<0.01)$. Other significant predictors of high depersonalisation were number of years as a MO prior to the training programme. The odds of depersonalisation increased: (i) 3 times if the study participant was younger than 35 years (OR 2.96; 95\% CI $0.55-5.38$; $p=0.02$ ); (ii) 3 times if the study participant had spent between 5 - 10 years as an MO (OR 3.12; 95\% CI $0.79-5.46$; $p<0.01$ ) and; (iii) 3.5 times if the study participant had spent $>10$ years as an MO (OR 3.56; 95\% CI 0.66 - 6.46; $p=0.02$ ). Year of study and relationship status were significant predictors of low personal accomplishment. The odds of personal accomplishment increased by 1.7 times if the participant was in their third year of study (OR 1.70; 95\% CI $0.01-3.82 ; p=0.04$ ) and decreased 2 times if the study participant was either married or single (OR 1.93; 95\% CI 0.68 - $3.8 ; p=0.04$ ).

\section{Discussion}

A study by Stodel et al. ${ }^{[3]}$ at the Red Cross War Memorial Children's Hospital in Cape Town, South Africa, reported high levels of burnout among junior doctors, resulting in a high level of work dissatisfaction and a desire to leave their job as soon as their current rotation ended. Similarly, a study of registrars and MOs in different medical specialties in Bloemfontein, showed significant levels of burnout in $32 \%$ of the participants studied ${ }^{[4]}$ Forty-eight percent suffered high levels of emotional exhaustion, $40 \%$ experienced high depersonalisation and $38 \%$ had low personal accomplishment. ${ }^{[4]}$ A caveat to the aforementioned study is that the authors did not report which medical specialties were studied, or whether there was a difference in outcome for the different specialties. ${ }^{[4]}$ A study by Heyworth et al. ${ }^{[8]}$ in an Accident and Emergency Unit in the UK reported burnout to be highest among junior doctors. Furthermore, senior registrars and consultants had lower stress levels and experienced a higher level of work satisfaction. This was attributed to better coping mechanisms, due to more work experience. $^{[8]}$ In support of this finding, a study of anaesthetists in SA showed that the more years spent working, the less the burnout experienced ${ }^{[5]}$ A qualitative study at Wayne State University School of Medicine, Detroit, USA, reported O\&G residents as having the highest prevalence of burnout among all medical specialties..$^{[9]}$ Similarly, Moradi et al. ${ }^{[10]}$ measured the prevalence of burnout in residency programmes, across a 31-year time period, that included 2509 participants. They showed a high level of burnout during the O\&G residency programme. While most residents matched the criteria for one category of burnout, there was, however, a high degree of burnout in $44 \%$ of the participants for all three subscales. ${ }^{[10]}$ In a survey study of O\&G residents across eight Spanish university hospitals, $64 \%$ of respondents showed high levels of depersonalisation, and $8 \%$ showed high levels of emotional exhaustion, $29 \%$ having high levels of both. ${ }^{[11]}$ The factors linked to increased burnout included being single and working long hours. ${ }^{[11]}$ Smith et al. ${ }^{[12]}$ conducted an online survey of 15800 participants reporting an estimated burnout rate of between $40 \%$ and $75 \%$ for all medical professionals and just over $50 \%$ among O\&G doctors in the field of dermatology were least affected, with a reported $43 \%$ overall burnout rate. ${ }^{[12]}$ Among O\&G, burnout was not limited to the USA, but was highly prevalent in the UK and Ireland. ${ }^{[12]}$ Residents and young physicians were at greater risk of burnout, having higher rates of suicidal ideation and depression than senior consultants or MOs with more years of experience. ${ }^{[12]}$ Furthermore, higher burnout rate was experienced in academic settings, ${ }^{[12]}$ leading to possible dissatisfaction in career choice and poor productivity in the workplace. Awareness of burnout symptoms, particularly at an early stage, is important in devising strategies to mitigate its effects. Stress and fatigue reduction techniques have been used, with positive outcomes. ${ }^{[12]}$

\section{Burnout and its subscales}

In the present study, high levels of emotional exhaustion were experienced by $85 \%$ of participants. High levels of depersonalisation were seen in $78 \%$ and just under $50 \%$ of the respondents experienced low personal accomplishment at work. Smith et al. ${ }^{[12]}$ demonstrated a $40-70 \%$ burnout rate across all medical specialties, with a burnout rate of just over $50 \%$ in O\&G. They noted that women have a higher burnout rate; however, burnout by subgroup was not assessed. ${ }^{[12]}$ Similarly, Van der Walt et al. ${ }^{[5]}$ showed an increased burnout rate amongst women. An increased burnout rate among women in the emotional exhaustion subgroup was observed, while rates for depersonalisation or low personal accomplishment were not statistically significant. ${ }^{[5]}$ In the present study, only $6 \%$ of participants experienced burnout in all three subgroups, lower than the $21 \%$ burnout rate reported among the participants. ${ }^{[5]}$ Our findings are comparable to the burnout rate experienced by private anaesthetists (8.1\%). ${ }^{[5]}$ Interestingly, the longer a doctor has worked in the department, the less the risk of burnout. ${ }^{[5]}$ Govardhan et al. ${ }^{[2]}$ showed a greater than $50 \%$ rate for burnout when only considering emotional exhaustion and depersonalisation $(20 \%$ lower than our findings). However, when all three parameters were combined, the rate was $13 \%$, almost double the rate found in our study. High rates of burnout were associated with decreased job satisfaction and increased rates of depression. ${ }^{[2]}$ Interestingly, personal accomplishment decreased for participants in the third year of their program, but not their fourth year, despite being close to completing their training programme. 
Personal accomplishment increased 2-fold when participants were in a stable relationship however, for married respondents ( $60 \%)$, this did not decrease the risk for low personal accomplishment. Although there are a few studies that have looked at burnout in residents over different specialties, ${ }^{[2-6,8-12]}$ none has examined the burnout rate for individual years of training. Zis et al. ${ }^{[6]}$ reported their participants were mostly 18 months before the completion of their training time, but that this was not statistically significant for burnout. In their study, factors associated with increased burnout included decreased supervisor support, work/ home demands and conflicts, lack of autonomy in the workplace, and decreased opportunities for professional development. ${ }^{[6]}$

\section{Study limitations}

Registrars and doctors may have been reluctant to participate in the questionnaires and surveys due to the possibility of personal identification, given the small sample size. Qualitative data that further interrogated the sentiments of the participants would have allowed a better interpretation of the quantitative data

\section{Conclusion}

Burnout is a significant problem among registrars in the $O \& G$ department at the University of the Witwatersrand. The risk factors associated with burnout should be confirmed in larger cohort studies, and where possible, interventions such as a wellness programme should be put in place to mitigate burnout risk. Working hours and after-hour supervision should be considered as possible causes for burnout. Support groups could also be useful. SADAG, in collaboration with Discovery Health, SA, have recently launched a Discovery Medical Student Helpline for students, interns and junior doctors. Hopefully, these services will be extended to registrars and senior doctors, when needed.

\section{Acknowledgements. None.}

Author contributions. GMB collected the data, analysed the results and wrote the manuscript. SJB and HAL supervised the work and contributed to the final version of the manuscript.

Funding. None.

Conflicts of interest. None.

1. Adinma J. Litigations and the obstetrician in clinical practice. Ann Med Health Sci Res 2016;6(2):74-79. https://doi.org/10.4103/2141-9248.181847

2. Govardhan LM, Pinelli V, Schnatz PF. Burnout, depression and job satisfaction in obstetrics and gynecology residents. Conn Med 2012;76(7):389-395.

3. Stodel JM, Stewart-Smith A. The influence of burnout on skills retention of junior doctors at Red Cross War Memorial Children's Hospital: A case study. S Afr Med J 2011;101(2):115-118. https://doi.org/10.7196/samj.4431

4. Sirsawy U, Steinberg W, Raubenheimer J. Levels of burnout among registrars and medical officers working at Bloemfontein public healthcare facilities in 2013. S Afr Fam Pract 2016;58(6):213-218 https://doi.org/10.1080/20786190.2016.1198088

5. van der Walt N, Scribante J, Perrie H. Burnout among anaesthetists in South Africa. S Afr J Anaesth Analg 2015;21(6):169-172. https://doi.org/10.1080/22201181.2015.1102798

6. Zis P, Artemiadis AK, Lykouri M, et al. Residency training: Determinants of burnout of neurology trainees in Attica, Greece. Neurology 2015;85(11):e81-84. https://doi.org/10.1212/ WNL.000000000000192

7. Maslach C, Jackson SE. The measurement of experienced burnout. J Organ Behav 1981;2(2):99-113 https://doi.org/10.1002/job.4030020205

8. Heyworth J, Whitley TW, Allison Jr EJ, Revickit DA, Heywortll J, Deqartnwnt E. Correlates of workrelated stress among consultants and senior registrars in accident and emergency medicine. Arch Emerg Med 1993;10(4):271-278. https://doi.org/10.1136/emj.10.4.271

9. Martini S, Arfken CL, Balon R. Comparison of burnout among medical residents before and after the implementation of work hours limits. Acad Psychiatry 2006;30(4):352-355. https://doi. org/10.1176/appi.ap.30.4.352

10. Moradi Y, Baradaran HR, Yazdandoost M, Atrak S, Kashanian M. Prevalence of burnout in residents of obstetrics and gynecology: A systematic review and meta-analysis. Med J Islam Repub Iran 2015;29(4):235.

11. Castelo-Branco C, Figueras F, Eixarch et al. Stress symptoms and burnout in obstetrics and gynaecology residents. BJOG 2006;114(1):94-98. https://doi.org/10.1111/j.1471-0528.2006.01155.x

12. Smith RP. Burnout in obstetricians and gynecologists. Obstet Gynecol Clin North Am 2017;44(2):297-310. https://doi.org/10.1016/j.ogc.2017.02.006

Accepted 5 November 2019. 